\title{
The impact of chromosomal microarray on clinical management: a retrospective analysis
}

\author{
Lindsay B. Henderson, PhD'1, Carolyn D. Applegate, MS, CGC1, Elizabeth Wohler, MS², \\ Molly B. Sheridan, PhD ${ }^{1}$, Julie Hoover-Fong, MD, PhD ${ }^{1}$ and Denise A.S. Batista, PhD ${ }^{1-3}$
}

\begin{abstract}
Purpose: Chromosomal microarray has been widely adopted as the first-tier clinical test for individuals with multiple congenital anomalies, developmental delay, intellectual disability, and autism spectrum disorders. Although chromosomal microarray has been extensively shown to provide a higher diagnostic yield than conventional cytogenetic methods, some health insurers refuse to provide coverage for this test, claiming that it is experimental and does not affect patients' clinical management.
\end{abstract}

Methods: We retrospectively reviewed the electronic medical records of all patients who had abnormal chromosomal microarray findings reported by our laboratory over a 3 -year period and quantified the management recommendations made in response to these results.

Results: Abnormal chromosomal microarray findings were reported for $12.7 \%$ of patients $(227 / 1,780)$. For patients with clinical follow-up notes available, these results had management implications for $54.5 \%$ of patients in the entire abnormal cohort (102/187) and for $42.1 \%$ of patients referred for isolated neurodevelopmental disorders (16/38). Recommendations included pharmacological treatment, cancerrelated screening or exclusion of screening, contraindications, and referrals for further evaluation.

Conclusion: These results empirically demonstrate the clinical utility of chromosomal microarray by providing evidence that management was directly affected for the majority of patients in our cohort with abnormal chromosomal microarray findings.

Genet Med advance online publication 13 March 2014

Key Words: autism; clinical management; clinical utility; chromosomal microarray; intellectual disability

\section{INTRODUCTION}

Genomic imbalances, although individually rare, are collectively a common cause of multiple congenital anomalies (MCAs), developmental delay/intellectual disability (DD/ID), and autism spectrum disorders (ASDs). Although karyotyping has been the gold standard for diagnosing individuals with these conditions, high-resolution G-banded karyotypes can detect only large, microscopically visible chromosomal changes $(>5-7 \mathrm{Mb}$ in size) and provide diagnoses in just $3-5 \%$ of cases. ${ }^{1}$ The advent of fluorescence in situ hybridization (FISH) increased the diagnostic yield by $\sim 2-3 \%$ in these patients by targeting known submicroscopic deletions and duplications; $;, 3$ however, many individuals exhibit features that cannot easily be ascribed to a well-known syndrome.

These technical limitations have been overcome through the development of chromosomal microarray (CMA), which is able to interrogate the entire genome at much higher resolution than conventional karyotyping and FISH. In addition to increased sensitivity for the detection of copy-number gains and losses, single-nucleotide polymorphism (SNP)based platforms also have the capability to reveal pathogenic regions of homozygosity attributed to uniparental disomy or parental consanguinity. Clinical implementation of CMA has greatly expanded since it gained the support of the American
College of Medical Genetics and Genomics as the first-tier diagnostic test for individuals with MCAs, DD/ID, and ASDs. ${ }^{4}$ Various studies have shown that CMA affords the identification of pathogenic copy-number changes (CNCs) in $15-20 \%$ of this patient population ${ }^{5-8}$ and to a lesser extent in individuals with epilepsy, schizophrenia, and other neuropsychiatric disorders. ${ }^{9-12}$ The diagnostic utility of CMA is exemplified by 22q11.2 deletion syndrome (classically, DiGeorge (OMIM 188400) or velocardiofacial (OMIM 192430) syndrome), which is caused by the most common known recurrent deletion. ${ }^{13}$ Patients manifesting hallmark features of this disorder, such as congenital heart defects, palatal abnormalities, and learning problems, have traditionally been tested by FISH. However, it has become evident through the use of CMA that $\sim 5 \%$ of patients with 22q11.2 deletion syndrome actually carry smaller, atypical deletions or duplications that cannot be detected by standard FISH. ${ }^{14}$ Furthermore, CMA frequently reveals 22q11.2 deletions in patients who are not suspected, based on their clinical features, to have this disorder but who are at risk for the other manifestations of this condition. This paradox reinforces the need for an unbiased, high-resolution diagnostic approach as the phenotypic and etiologic diversity of "classical" syndromes expands and as new syndromes are continually described. 
US health insurance providers are inconsistent in their coverage (or lack of coverage) of genetic testing and genetic counseling services. Despite numerous reports on the diagnostic utility of $\mathrm{CMA}^{4,5,15}$ and its wide acceptance by the clinical genetics community, some health insurance companies do not provide reimbursement for this test, particularly for patients with isolated or nonsyndromic DD/ID and ASDs. The Secretary's Advisory Committee on Genetics, Health, and Society concluded that "coverage and reimbursement are critical to ensuring appropriate access to genetic tests and services and their integration into clinical practice." ${ }^{16}$ Health insurance providers make coverage decisions based on the quality of existing clinical evidence and whether the test will directly influence disease management and thereby result in improved net health outcomes. Moreover, collection and evaluation of direct evidence showing improved health outcomes after reaching a diagnosis is difficult and may not always be possible. ${ }^{17,18} \mathrm{~A}$ genetic diagnosis allows physicians to choose proper treatments and establish likely prognosis. Although this is true for many disorders, such as inborn errors of metabolism and familial cancer syndromes, there is a paucity of empirical data demonstrating the influence of CMA findings on the care of patients with other genetic conditions.

Two recent studies have correlated abnormal CMA findings with predicted clinical impact. Ellison et al. ${ }^{15}$ reviewed data from 46,298 patients tested by CMA and assessed the frequency of pathogenic abnormalities associated with any of 151 disorders they had deemed to be clinically actionable. The authors estimated that $35 \%$ of pathogenic abnormalities found in their population would warrant clinical follow-up. More recently, Riggs et al. ${ }^{19}$ queried 28,526 cases and 146 clinically actionable phenotypes in the International Standards for Cytogenomics Arrays Consortium database and concluded that $46 \%$ of all pathogenic or likely pathogenic CNCs submitted $(1,908 / 4,125)$, or $7 \%$ of all cases in the database, would potentially have implications for clinical management. Despite these two large data sets, little empiric data have been published to assess the extent to which CMA influences clinical management based on the health-care providers' actual recommendations. Coulter et al..$^{20}$ reviewed CMA reports from 1,792 patients, of whom 235 had an abnormal or possibly abnormal result. The authors determined that the actual rate of clinical action based on CMA findings was 54\% for abnormal and $34 \%$ for possibly abnormal CNCs, or $5.1 \%$ of all cases tested. To further evaluate the direct impact of CMA findings on patients' clinical management in an independent population, we have undertaken a retrospective review of all abnormal cases reported by our laboratory over a 3-year period.

\section{Subjects and CNC analysis}

\section{MATERIALS AND METHODS}

We reviewed the results of 1,780 consecutive cases received in our laboratory for CMA analysis between August 2009 and August 2012. Cases were predominantly from the Johns Hopkins Hospital and Kennedy Krieger Institute, Baltimore, MD, but 54 (3.0\%) were from other local hospitals. This study was approved by the Johns Hopkins Medicine Institutional Review Board.

All samples were analyzed using a high-resolution SNP microarray platform, either the HumanQuad610 BeadChip or Human Omni 1M BeadChip; analysis was performed according to the manufacturer's (Illumina, San Diego, CA) protocol. CNCs were interpreted in accordance with previously established guidelines for assessing pathogenicity ${ }^{5,21}$ and our own laboratory-developed practices and internal databases of abnormal and benign variants. In general, $\mathrm{CNCs}$ were reported as abnormal (i.e., pathogenic or possibly pathogenic) if they overlapped known microdeletion or microduplication syndrome regions, encompassed or interrupted disease-associated genes (e.g., OMIM disease-causing genes; http://www.omim.org/), or included numerous genes that were not found in public databases of presumably healthy individuals (http://cnv.chop.edu/; http:// dgv.tcag.ca/). ${ }^{22,23}$ Large regions of homozygosity ( $\left.>10 \mathrm{Mb}\right)$ confined to a single chromosome were also interpreted as abnormal because this finding is suggestive of uniparental disomy, a mechanism known to cause disorders of imprinting and uncover recessive alleles. Four such cases were subsequently confirmed to represent uniparental disomy by methylation studies and/or comparison to parental SNP array results, and another case was already known to harbor an apparently homozygous mutation causing a recessive disorder. Heterozygous changes that affected recessive disease-causing genes were not classified as abnormal, however; nor were partial gene duplications that would have unknown effects on transcription. CNCs larger than our size cutoffs ( $>200 \mathrm{~kb}$ for deletions and $>500 \mathrm{~kb}$ for duplications) were reported as variants of uncertain significance if they did not fulfill the above criteria and had never or rarely been reported in healthy individuals (frequency: $<0.2 \%$ ). CNCs were considered benign if they did not involve disease-causing genes, were frequently found in the databases of healthy individuals cited above (frequency $>0.5 \%$ ), and were seen in $\geq 1 \%$ of our patient population. These benign variations were typically not reported. For the purposes of this study, only subjects with abnormal CMA findings were included in our analyses. Abnormal parental or sibling follow-up testing was not included.

Among the subjects studied were 41 patients with known cytogenetic abnormalities previously diagnosed by karyotype, FISH, or multiplex ligation-dependent probe amplification. Although these were not "new" diagnoses, we felt that their inclusion was warranted because CMA can provide refinement of chromosomal break points and more precise determination of genes encompassed or excluded by the known abnormality. Furthermore, CMA may uncover additional CNCs not detected by the original diagnostic method, particularly in cases in which patients' clinical features did not seem fully explained by the known chromosome abnormality.

\section{Clinical data collection and evaluation of the impact of CMA findings on management}

Patients' clinic and genetic counseling notes within our electronic medical record systems were reviewed using a 
standardized data collection template developed by the study team members to ensure objective and uniform data collection.

CMA findings were considered to have directly impacted patients' clinical management if they prompted physicians or genetic counselors to recommend further action such as pharmacologic treatment, cancer-related screening or surveillance, contraindications, or additional clinical evaluation by imaging, laboratory tests, or referrals to other specialists. Instances in which the CMA findings ruled out the involvement of a cancer gene in patients with a previously identified cytogenetic abnormality were also considered to have altered management, whereby the need for cancer screening was eliminated. We strived to include in our analysis only those recommendations made as a direct outcome of the CMA findings. Specialist evaluations, imaging, laboratory testing, and treatments completed prior to CMA were not included, nor did we include clinical recommendations made after CMA for other reasons not specific to the abnormal CMA findings. Although necessary for elucidating the chromosomal abnormality and determining recurrence risk, recommendations for additional cytogenetic studies such as karyotype, FISH, or parental testing were not counted as changes to patients' management. Clinical followup time ranged from 13 months to 3 years, depending on when patients underwent CMA testing during the study period.

\section{RESULTS}

\section{Patient demographics}

We screened 1,780 patients for CMA in our laboratory over a 3-year period between 2009 and 2012. Of these, 227 patients (12.7\%) had abnormal CMA findings, 240 (13.5\%) had variants of uncertain significance, and 1,313 (73.8\%) had normal findings at the level of resolution analyzed (Figure 1). To assess how CMA findings affected the medical management of patients, we limited our retrospective analysis to the subset of patients with abnormal CMA findings. The majority of these cases $(n=148$; $65.2 \%$ ) had their SNP array ordered by clinical geneticists at the Johns Hopkins Hospital, and about a quarter of these cases $(n=35)$ were neonatal intensive care unit consults. Neurologists at the Kennedy Krieger Institute ordered 61 additional CMAs

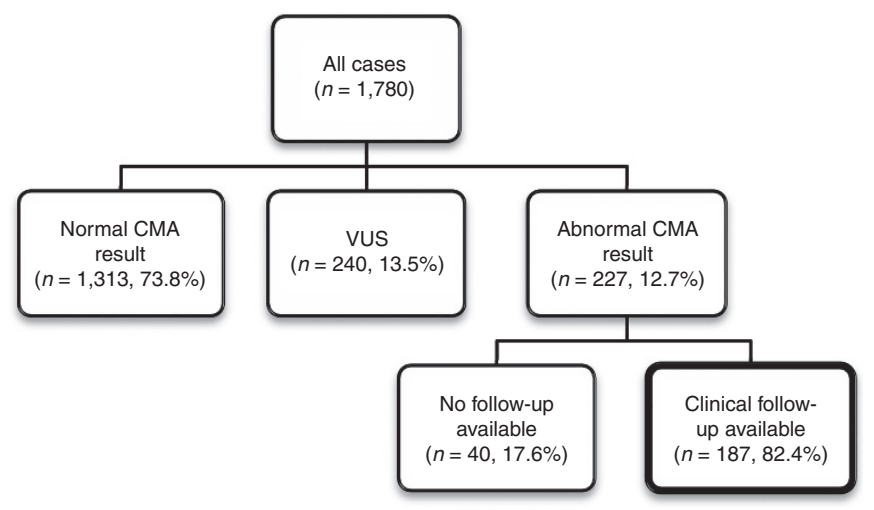

Figure 1 Breakdown of chromosomal microarray (CMA) results and clinical follow-up among cases collected over a 3-year period. VUS, variants of uncertain significance. that were found to be abnormal (26.9\%), and a smaller number of abnormal CMAs were ordered by other specialists within the Johns Hopkins Hospital ( $n=8 ; 3.5 \%)$ or from other community hospitals $(n=10 ; 4.4 \%)$. The median patient age was 4.7 years, and more males had abnormal CMA findings than females (131 (57.7\%) vs. 96 (42.3\%), respectively). The elevated male to female ratio in cases with abnormal results was reflective of the sex distribution observed in all other cases tested in our cohort $(n=1,553)$, i.e., those who had normal CMA findings or variants of uncertain significance (948 males (61.0\%) vs. 605 females $(39.0 \%) ; P=0.34)$. A listing of the genetic abnormalities identified in this cohort can be found in Supplementary Tables S1-S3 online.

The indications for performing CMA in those patients found to have an abnormal array result are shown in Table 1. Almost two-thirds of patients (63.4\%) presented with some form of neurodevelopmental disorder, including DD/ID, seizures, ASD, or a combination of these. The majority of patients had congenital anomalies or dysmorphic features (68.7\%). Failure to thrive and short stature were also common indications for testing. Overall, there were 469 indications among 227 patients, demonstrating that on average patients had at least two major clinical features that warranted physicians to order CMA testing.

To determine the effect of abnormal CMA findings on the clinical management of patients, we quantified the number of recommendations made by physicians and genetic counselors in response to these results after a minimum follow-up period of 13 months. Clinical follow-up notes referencing the abnormal CMA findings were available for 187 individuals $(82.4 \%)$ after the result was given. For the remaining fraction of patients, clinical follow-up notes specific to the abnormal CMA

Table 1 List of 469 testing indications for 227 patients with abnormal CMA findings

\begin{tabular}{lc} 
Clinical feature & $\begin{array}{c}\text { Number of } \\
\text { patients (\%) }\end{array}$ \\
\hline Neurodevelopmental disorder & $150(66.1)$ \\
Developmental delay & $106(46.7)$ \\
Learning disability or behavioral or psychiatric disorder & $30(13.2)$ \\
Seizures & $28(12.3)$ \\
Intellectual disability & $25(11.0)$ \\
Autism spectrum disorder & $24(10.6)$ \\
Congenital anomalies & $117(51.5)$ \\
Isolated anomaly & $70(30.8)$ \\
Multiple anomalies & $47(20.7)$ \\
Dysmorphic features & $76(33.5)$ \\
Abnormal growth & $35(15.4)$ \\
Failure to thrive & $22(9.7)$ \\
Short stature & $14(6.2)$ \\
Hypotonia & $27(11.9)$
\end{tabular}

CMA, chromosomal microarray.

asum of percentages exceeds 100 because some patients had more than one referral reason ( 2.1 on average). 
Table 2 Summary of recommendations made by healthcare providers in response to abnormal CMA findings

Number of patients $(\%)^{\mathrm{a}}$

\begin{tabular}{|c|c|c|}
\hline \multirow[b]{2}{*}{ Recommendation } & \\
\hline & $\begin{array}{l}\text { All indications } \\
\text { for testing } \\
(n=187)\end{array}$ & $\begin{array}{l}\text { Neurodevelopmental } \\
\text { indications only } \\
(n=38)^{\mathrm{b}}\end{array}$ \\
\hline Clinical action & $24(12.8)$ & $3(7.9)$ \\
\hline Pharmacologic treatment & $6(3.2)$ & - \\
\hline $\begin{array}{l}\text { Angiotensin receptor } \\
\text { blocker }\end{array}$ & $1(0.5)$ & - \\
\hline Cysteamine & $1(0.5)$ & - \\
\hline Growth hormone & $3(1.6)$ & - \\
\hline Testosterone & $1(0.5)$ & - \\
\hline $\begin{array}{l}\text { Cancer-related screening or } \\
\text { surveillance }\end{array}$ & $11(5.9)$ & $3(7.9)$ \\
\hline APC deletion & $3(1.6)$ & $1(2.6)$ \\
\hline VHL deletion & $2(1.1)$ & $1(2.6)$ \\
\hline MLH1 deletion & $1(0.5)$ & $1(2.6)$ \\
\hline NSD1 deletion & $1(0.5)$ & - \\
\hline SMARCB1 deletion & $1(0.5)$ & - \\
\hline CREBBP deletion & $1(0.5)$ & - \\
\hline $4 q 21$ deletion & $1(0.5)$ & - \\
\hline Mosaic monosomy 7 & $1(0.5)$ & - \\
\hline $\begin{array}{l}\text { Cancer-related screening } \\
\text { avoided }\end{array}$ & $3(1.6)$ & - \\
\hline$A P C$ not deleted & $2(1.1)$ & - \\
\hline VHL not deleted & $1(0.5)$ & - \\
\hline Contraindication & $4(2.1)$ & - \\
\hline $\begin{array}{l}\text { Avoid excess calcium and } \\
\text { vitamin D }\end{array}$ & $3(1.6)$ & - \\
\hline Avoid live viral vaccines & $1(0.5)$ & - \\
\hline Further clinical evaluation & $95(50.8)$ & $14(36.8)$ \\
\hline Specialist referral & $84(44.9)$ & $12(31.6)$ \\
\hline Cardiology & $38(20.3)$ & $8(21.1)$ \\
\hline Audiology & $36(19.3)$ & $1(2.6)$ \\
\hline Ophthalmology & $33(17.7)$ & $2(5.3)$ \\
\hline Immunology & $18(9.6)$ & - \\
\hline $\begin{array}{l}\text { Neurology (includes brain } \\
\text { MRI) }\end{array}$ & $13(7.0)$ & $2(5.3)$ \\
\hline Endocrinology & $11(5.9)$ & $1(2.6)$ \\
\hline Otolaryngology & $9(4.8)$ & - \\
\hline Other & $15(8.0)$ & $1(2.6)$ \\
\hline Imaging $^{d}$ & $38(20.3)$ & $6(15.8)$ \\
\hline Laboratory testing & $29(15.5)$ & $2(5.3)$ \\
\hline $\begin{array}{l}\text { Overall impact on clinical } \\
\text { management }\end{array}$ & $102 / 187(54.5)$ & $16 / 38(42.1)$ \\
\hline
\end{tabular}

MRI, magnetic resonance imaging.

alncludes only those patients with abnormal chromosomal microarray (CMA) results and clinical follow-up available; percentages are not additive because some patients had multiple recommendations (see Table 3). ${ }^{b}$ Includes only those patients with isolated neurodevelopmental indications, including developmental delay, learning disabilities, behavioral or psychiatric disorders, seizures, intellectual disability, and/or autism spectrum disorders. Includes referrals for sleep study, orthopedics, nephrology, gastroenterology, pulmonology, hematology, and muscular dystrophy clinic. Includes renal or abdominal ultrasound, $\mathrm{X}$-ray, and skeletal survey. finding were not available in the electronic medical records. Recommendations deemed to be a direct consequence of the abnormal CMA findings were categorized as clinical action: pharmacologic treatment, cancer-related screening, exclusion of cancer screening, or contraindications; or as referrals for further evaluation: evaluations by other specialists, imaging studies, or laboratory testing (Table 2).

\section{Pharmacologic treatment}

Once a diagnosis is established, patients may benefit from therapies that have been shown to improve outcomes in others with the same genetic condition. We identified six patients (3.2\%) for whom specific drug therapy was recommended in response to the diagnosis made by CMA. The first case was a 5 -year old evaluated by the genetic medicine service due to history of a heart defect and enlarged aortic root, $\mathrm{DD}$, hypotonia, and joint hypermobility. CMA revealed a $1.4 \mathrm{Mb}$ deletion on chromosome 15 encompassing FBN1. Haploinsufficiency of this gene is associated with mild-to-classical presentation of Marfan syndrome (OMIM 154700). ${ }^{24}$ Given the patient's CMA finding and cardiac features consistent with a diagnosis of Marfan syndrome, treatment was initiated with an angiotensin receptor blocker therapy to control aortic growth. Within months, this therapy successfully normalized the rate of growth of the patient's aortic root. Another case was a child who presented with multiple electrolyte abnormalities and was clinically diagnosed with Fanconi syndrome given her history of failure to thrive, polydipsia, polyuria, and hypophosphatemic rickets. Cystinosis (OMIM 219800) was highly suspected, but because biochemical studies returned equivocal results and insurance issues impeded approval for gene-specific testing, CMA was subsequently ordered to test for the most common mutation underlying this disorder, a $57 \mathrm{~kb}$ partial deletion of CTNS. ${ }^{25}$ The array revealed a homozygous deletion of this segment, thereby confirming the diagnosis of cystinosis. This allowed the patient to immediately begin treatment with cysteamine, a compound that helps to preserve renal function and improve growth in patients with cystinosis. ${ }^{26}$ Furthermore, growth hormone treatment was suggested for three children with short stature attributed to their genetic abnormality detected by CMA (deletion of SHOX, deletion of 18q, and tetrasomy for 18p). In children for whom growth hormone treatment is appropriate, early intervention with this therapy has been shown to maximize their growth potential. ${ }^{27}$ Finally, testosterone treatment was recommended for a patient diagnosed by array with Klinefelter syndrome once he reaches puberty.

\section{Cancer-related screening}

The identification of CNCs associated with a predisposition to cancer is often an unanticipated CMA finding ${ }^{15}$ and can have significant bearing on patients' management. Among our abnormal cases, there were 11 patients $(5.9 \%)$ whose array results involved a gene or region linked to cancer risk and prompted further clinical evaluation. In all but one of these patients, the involvement of a cancer predisposing gene was an incidental 
finding, as their indications for testing were DD/ID, seizures, dysmorphic features, and/or MCA. We identified three patients with deletions encompassing APC, two with deletion of $V H L$, and one with deletion of $M L H 1$. These genes underlie familial adenomatous polyposis (OMIM 175100), Von Hippel-Lindau syndrome (OMIM 193300), and Lynch syndrome (OMIM 609310), respectively, all of which are well-described cancer syndromes requiring rigorous screening according to published guidelines. ${ }^{28,29}$

Screening was also warranted in five patients with other diagnoses having cancer as a reported feature: (i) a child with failure to thrive and recurrent infections was found to have mosaic monosomy 7 on CMA analysis of blood. Due to the association of this finding with risk for myelodysplastic syndrome, bone marrow cytogenetic studies were conducted and revealed the same abnormal karyotype. The patient subsequently underwent a bone marrow transplant to prevent progression to leukemia. (ii) A newborn with MCA and a terminal 5q deletion encompassing NSD1 was recommended to have regular alpha-fetoprotein testing and abdominal ultrasounds because mutation of this gene has been reported in some cases of Beckwith-Wiedemann syndrome (OMIM 130650), which has an increased risk for the development of Wilms tumors. ${ }^{30}$ (iii) A dysmorphic child with DD and hypotonia was found to have an interstitial 4q21 deletion and was recommended to have alpha fetoprotein screening for hepatoblastoma due to the presentation of this tumor type in another patient with deletion of the same region. ${ }^{31}$ (iv) CMA detected an intragenic CREBBP deletion in an infant with bilateral thumb deformities and DD, consistent with a diagnosis of Rubinstein-Taybi syndrome (OMIM 180849). Because various malignancies have been reported in this syndrome and the patient was found to have an elevated white blood cell count, a consultation with an oncologist was arranged. (v) A child referred for CMA because of seizures and cardiac abnormalities was shown to have a distal 22q11.2 deletion disrupting SMARCB1. The patient was recommended to have a renal ultrasound and a complete dermatological examination based on knowledge that deletion of this gene predisposes patients to soft-tissue rhabdoid tumors.

Notably, an indication of cancer was evident for only one patient, a newborn female with a large prenatally identified hepatoblastoma. Because the infant met some diagnostic criteria for Beckwith-Wiedemann syndrome, methylation testing and CMA were ordered to examine for this disorder. Of interest, CMA detected an interstitial 5q22.2 deletion encompassing $A P C$, establishing a diagnosis of familial adenomatous polyposis. $^{32}$ Although this disorder was included in the differential diagnosis as a cause for hepatoblastoma, it was not learned until later that the proband's mother had an extensive maternal family history of colon cancer. Furthermore, the mother was also noted to have thyroid nodules early in the pregnancy, which were diagnosed postpartum as papillary thyroid carcinoma. She was subsequently tested by CMA and found to harbor the same deletion as her child. These results had enormous impact not only on the medical management of both mother and child but potentially on other family members who would also be at risk for developing familial adenomatous polyposis. Furthermore, the mother's result provided valuable information regarding recurrence risk for future pregnancies.

\section{Cancer screening avoided}

Three patients with abnormalities originally identified by karyotype were referred for CMA to more precisely delineate the break points and determine whether their abnormality encompassed a cancer gene known to be in the vicinity. CMA excluded the involvement of APC in two patients and that of $V H L$ in another patient, thus eliminating the need for costly lifelong tumor screening.

\section{Contraindications}

We also assessed whether health-care providers indicated any medical contraindications as a consequence of abnormal CMA findings. Patients diagnosed with Williams syndrome (OMIM 194050) are advised against taking multivitamins with extra calcium or vitamin $\mathrm{D}$, given that hypercalcemia is a known potential clinical complication of this disorder. In our patient population, seven patients were diagnosed with Williams syndrome, and the clinical notes of three of these patients specifically mentioned this contraindication, whereas the records of the remaining four did not. Although all seven subjects were included in our analysis, only the explicitly documented recommendations were tallied. However, through discussions with our clinical genetics specialists and genetic counselors, it was emphasized that this recommendation is standard of care for Williams syndrome and would not be made without the cytogenetic diagnosis.

Immune deficiency is a feature present in $77 \%$ of cases with 22q11.2 deletion syndrome. ${ }^{33}$ Children with a 22q11.2 deletion should be cautioned against immunization with live viral vaccines until immune status is clarified because these may be unsafe in patients with immunodeficiency. During the study period, we diagnosed 22q11.2 deletion in 22 individuals, of whom 11 were under 5 years of age. All these subjects were included in our denominator of patients with abnormal array results; however, the clinical notes of only one of these patients explicitly mentioned this contraindication. Although not counted in our analysis, it is important to note that a similar recommendation regarding vaccination may also be appropriate for the other patients with 22q11.2 deletions, with reevaluation of immunologic status prior to administration of live viral vaccines.

\section{Referrals for further clinical evaluation}

Further clinical evaluation was advised for more than half of all patients ( $n=95 ; 50.8 \%)$ with abnormal CMA findings and clinical follow-up available (Table 2). Many patients were referred to one or more medical specialists $(n=84 ; 44.9 \%)$. In addition, imaging and laboratory testing were recommended for $20.3 \%$ $(n=38)$ and $15.5 \%(n=29)$ of cases, respectively. These recommendations were not mutually exclusive, as patients may have 
Table 3 Distribution of clinical recommendations among patients with abnormal CMA findings

\begin{tabular}{|lc} 
Number of recommendations per patient & $\begin{array}{l}\text { Number of } \\
\text { patients (\%) }\end{array}$ \\
\hline 0 & $85(45.5)$ \\
\hline 1 & $40(21.4)$ \\
\hline 2 & $18(9.6)$ \\
\hline 3 & $15(8.0)$ \\
\hline 4 & $29(15.5)$ \\
\hline Total (264 recommendations) & 187 \\
\hline
\end{tabular}

CMA, chromosomal microarray.

had any combination of specialist referrals, imaging, and laboratory tests prescribed as part of their follow-up.

In total, 264 medical recommendations were made by medical professionals in response to 187 abnormal CMA findings, yielding an average of 1.4 recommendations per patient. The distribution of recommendations per patient is shown in Table 3.

Overall, $54.5 \%(n=102)$ of patients were given at least one recommendation. These findings demonstrate that CMA testing influenced clinical management for the majority of all patients with abnormal results in our cohort.

\section{Impact of CMA on management of patients with isolated neurodevelopmental indications}

In addition to quantifying changes in management among individuals with any indications for testing, we assessed clinical recommendations made for the subgroup of patients with isolated neurodevelopmental indications, including DD/ID, ASDs, learning disabilities, behavioral or psychiatric disorders, and/or seizures (Table 2). These individuals were not reported to have dysmorphic features, congenital anomalies, or growth abnormalities. Fifty subjects out of 227 with abnormal CMA findings $(22.0 \%)$ had purely neurodevelopmental indications for testing, and 38 of these (76.0\%) had follow-up records available. We found that direct clinical action or further evaluation was recommended for $42.1 \%$ patients (16/38), including three individuals with deletions of cancer susceptibility genes, revealing that abnormal CMA findings substantially impacted the management of patients with seemingly isolated neurological phenotypes in our population.

\section{DISCUSSION}

An accurate genetic diagnosis is paramount for assessing a patient's risk for developing certain complications, estimating prognosis, and developing a plan for disease management, including choice of appropriate therapy and environmental modifications to maximize an individual's health. A multitude of data show that the diagnostic yield of CMA is superior to traditional cytogenetic analysis by karyotype. Nevertheless, this evidence has not been sufficient to convince all health insurance providers to offer coverage for this test. The typically stated reason is that CMA is experimental and does not affect patient management.
To establish the degree to which CMA influenced the clinical management of our patient population, we retrospectively reviewed the medical records of 187 patients who had an abnormal CMA findings reported by our laboratory and had clinical follow-up notes available. Our approach was to specifically quantify only the management recommendations that were made after CMA testing and as a direct response to the abnormal result. We therefore did not count any clinical evaluations that had already been performed or recommended on account of patients' clinical presentation. For example, some physicians recommended an echocardiogram to rule out structural anomalies at the time the CMA was ordered. In these instances, the echocardiogram was not counted as a management recommendation even if an echocardiogram is recommended by diseasespecific surveillance guidelines. In addition, we did not include recommendations that were not explicitly recorded in the medical records. However, it should be noted that for at least 25 additional patients (4 patients with Williams syndrome and 21 with 22q11.2 deletion syndromes), specific clinical actions would be warranted. In comparison, the studies by Ellison et al. ${ }^{15}$ and Riggs et al. ${ }^{19}$ considered the theoretical management implications of an abnormal CMA diagnosis, whereas only the study by Coulter et al..$^{20}$ compiled all actual clinical recommendations made by patients' health-care providers.

Our analysis demonstrates that direct clinical action was recommended for $12.8 \%(24 / 187)$ of patients, and about half of the patients $(95 / 187)$ were referred for further testing to assess features known to be associated with their diagnosis. It would be expected that specialist referrals might lead to an improvement in the quality of patients' health by identifying and managing other problems that were not evident prior to the diagnosis. Therefore, CMA findings directly influenced clinical management in the majority of abnormal cases $(54.5 \%, 102 / 187)$ and, at minimum, $5.7 \%$ of our entire cohort $(102 / 1,780)$. It should be noted that the degree to which CMA alters management may vary depending on the phenotypes ascertained in the study population. To further explore this, we evaluated cases with only nonsyndromic neurodevelopmental indications $(22 \%$ of our abnormal cohort; 50/227) and found that $42.1 \%$ of these (16/38 with clinical follow-up available) received management recommendations. This rate was slightly lower than, but not statistically different from, the rate of recommendations made in our entire cohort of cases with abnormal results $(P=0.42)$. Importantly, this finding supports the clinical utility of CMA in individuals with isolated DD, ID, ASDs, and other neurodevelopmental phenotypes.

Although our assessment of the degree to which CMA influences clinical management is comparable to that of the aforementioned studies, our results are likely underestimated for several reasons. First, we were limited by the information available in the electronic medical records. Perhaps some recommendations and referrals that were discussed in the clinic or over the phone were not electronically documented, or patients may have received additional instruction from healthcare providers outside of our institutions, and thus we would 
not have access to these records. Second, we included 41 patients with previously diagnosed cytogenetic abnormalities. In most cases, these patients likely would have already received some recommendations for their management, although utilization of this technology may have produced additional recommendations and afforded a new opportunity to reinforce prior information. Most importantly, CMA would have detected all of these abnormalities had it been done as the firsttier test, thereby avoiding the additional cost of less-precise tests. Another caveat to our study design is that more recently accrued patients had a shorter follow-up period than those who were tested earlier. The earliest tested patients had up to 3 years to return for follow-up appointments, whereas the most recently accrued subjects had only 13 months and therefore less opportunity for additional recommendations to be made. Finally, our study did not incorporate cases reported to have variants of uncertain significance, and at least some fraction of these might have incited additional clinical action. As new microdeletion and duplication syndromes are described and known syndromes are better characterized through highquality CMA and careful phenotyping, this knowledge will help guide clinical management of and predict prognosis for affected individuals.

Achieving a genetic diagnosis yields numerous benefits. First, making a diagnosis allows recurrence risk to be estimated, enabling parents and other relatives to make informed decisions about future pregnancies. The advantages of knowing a patient's diagnosis can extend beyond the affected individual. Follow-up parental testing occasionally reveals that a parent carries the same genetic abnormality as his/her child, despite being apparently unaffected or less obviously affected. In this case, the same clinical recommendations made for the affected child may potentially be applicable to the parent as well. Similarly, if other family members are also at risk, the necessary steps can be taken to coordinate genetic testing and medical management of these individuals. Furthermore, better anticipatory guidance can also help facilitate acquisition of appropriate school placement as well as educational, occupational, and medical resources. For many families, a diagnosis means the end of a long and expensive "diagnostic odyssey." Parents may find comfort in finally learning the cause of their child's disabilities and being able to connect with other families through support groups. A diagnosis also allows parents to consider estate planning and make plans for their family's long-term care needs. All of these benefits afforded by achieving a genetic diagnosis, although highly valuable, were not accounted for in our study.

The potential health-care benefit of CMA in individuals with dysmorphic features, MCA, DD/ID, and ASDs is clear, as evidenced by the studies published by Ellison et al. ${ }^{15}$, Riggs et al. ${ }^{19}$, and Coulter et al. ${ }^{20}$, which showed that abnormal array results frequently have implications for patients' medical management. This is further confirmed by our observation that CMA influenced clinical care in more than half of our cohort with abnormal results. The unique feature of our study, however, is the fact that we were able to quantify the precise type and range of clinical intervention that occurred for all patients tested in a single laboratory in a sequential manner over 3 years. These data have applicability to large-scale populations with dysmorphisms, DD/ID, and/or ASDs studied by insurance companies and public health officials making medical and policy decisions about the clinical utility of CMA. Clearly, the next step is to carry out prospective studies to determine if medical management recommendations were followed, how much this cost, and what impact it had on health outcomes. In the meantime, we, as health-care providers, have an obligation to educate insurers and public policy makers about what we already know to be true for our patients and their families with a new chromosomal diagnosis-that an early and efficient diagnosis by CMA reduces the cost and stress of a long and fruitless workup, improves anticipatory guidance, and empowers the family with accurate genetic counseling.

\section{SUPPLEMENTARY MATERIAL}

Supplementary material is linked to the online version of the paper at http://www.nature.com/gim

\section{ACKNOWLEDGMENTS}

The authors thank the staff at the Cytogenetics and Microarray Laboratory at the Kennedy Krieger Institute. This work was partially supported by National Institutes of Health grant 5P30 HD024061-23 to the Intellectual and Developmental Disabilities Research Center.

\section{DISCLOSURE}

The authors declare no conflict of interest.

\section{REFERENCES}

1. Shevell M, Ashwal S, Donley D, et al.; Quality Standards Subcommittee of the American Academy of Neurology; Practice Committee of the Child Neurology Society. Practice parameter: evaluation of the child with global developmental delay: report of the Quality Standards Subcommittee of the American Academy of Neurology and The Practice Committee of the Child Neurology Society. Neurology 2003;60:367-380.

2. Ravnan JB, Tepperberg JH, Papenhausen P, et al. Subtelomere FISH analysis of 11688 cases: an evaluation of the frequency and pattern of subtelomere rearrangements in individuals with developmental disabilities. J Med Genet 2006;43:478-489.

3. Rauch A, Hoyer J, Guth S, et al. Diagnostic yield of various genetic approaches in patients with unexplained developmental delay or mental retardation. Am J Med Genet A 2006;140:2063-2074.

4. Manning M, Hudgins L; Professional Practice and Guidelines Committee Array-based technology and recommendations for utilization in medical genetics practice for detection of chromosomal abnormalities. Genet Med 2010;12:742-745.

5. Miller DT, Adam MP, Aradhya S, et al. Consensus statement: chromosomal microarray is a first-tier clinical diagnostic test for individuals with developmental disabilities or congenital anomalies. Am J Hum Genet 2010;86:749-764.

6. Cooper GM, Coe BP, Girirajan S, et al. A copy number variation morbidity map of developmental delay. Nat Genet 2011;43:838-846.

7. Sebat J, Lakshmi B, Malhotra D, et al. Strong association of de novo copy number mutations with autism. Science 2007;316:445-449.

8. Marshall CR, Noor A, Vincent JB, et al. Structural variation of chromosomes in autism spectrum disorder. Am J Hum Genet 2008;82:477-488.

9. Cook EH Jr, Scherer SW. Copy-number variations associated with neuropsychiatric conditions. Nature 2008;455:919-923.

10. Stefansson H, Rujescu D, Cichon S, et al.; GROUP. Large recurrent microdeletions associated with schizophrenia. Nature 2008;455:232-236. 
11. Purcell SM, Wray NR, Stone JL, et al. Common polygenic variation contributes to risk of schizophrenia and bipolar disorder. Nature 2009;460:748-752.

12. Mefford HC, Muhle H, Ostertag P, et al. Genome-wide copy number variation in epilepsy: novel susceptibility loci in idiopathic generalized and focal epilepsies. PLoS Genet 2010;6:e1000962.

13. Kaminsky EB, Kaul V, Paschall J, et al. An evidence-based approach to establish the functional and clinical significance of copy number variants in intellectual and developmental disabilities. Genet Med 2011;13:777-784.

14. Verhagen JM, Diderich KE, Oudesluijs $G$, et al. Phenotypic variability of atypical 22q11.2 deletions not including TBX1. Am J Med Genet A 2012;158A:24122420.

15. Ellison JW, Ravnan JB, Rosenfeld JA, et al. Clinical utility of chromosomal microarray analysis. Pediatrics 2012;130:e1085-e1095.

16. Department of Health and Human Services. Coverage and Reimbursement of Genetic Tests and Services: Report of the Secretary's Advisory Committee on Genetics, Health, and Society. http://oba.od.nih.gov/oba/sacghs/reports/ CR_report.pdf.

17. The Lewin Group. The Value of Diagnostics: Innovation, Adoption and Diffusion into Health Care. http://www.lewin.com/publications/publication/237/. Accessed 25 June 2013.

18. The Lewin Group. The Value of Laboratory Screening and Diagnostic Tests for Prevention and Health Care Improvement. http://www.lewin.com/publications/ publication/388/. Accessed 25 June 2013.

19. Riggs $E$, Wain $K$, Riethmaier $D$, et al. Chromosomal microarray impacts clinical management. Clin Genet 2014;85:147-153.

20. Coulter ME, Miller DT, Harris DJ, et al. Chromosomal microarray testing influences medical management. Genet Med 2011;13:770-776.

21. Lee C, lafrate AJ, Brothman AR. Copy number variations and clinical cytogenetic diagnosis of constitutional disorders. Nat Genet 2007;39(suppl 7): S48-S54.

22. Zhang J, Feuk L, Duggan GE, Khaja R, Scherer SW. Development of bioinformatics resources for display and analysis of copy number and other structural variants in the human genome. Cytogenet Genome Res 2006;115:205-214.

23. Shaikh TH, Gai X, Perin JC, et al. High-resolution mapping and analysis of copy number variations in the human genome: a data resource for clinical and research applications. Genome Res 2009;19:1682-1690.

24. Hilhorst-Hofstee $Y$, Hamel BC, Verheij JB, et al. The clinical spectrum of complete FBN1 allele deletions. Eur J Hum Genet 2011;19:247-252.

25. Shotelersuk V, Larson D, Anikster Y, et al. CTNS mutations in an American-based population of cystinosis patients. Am J Hum Genet 1998;63:1352-1362.

26. Gahl WA, Reed GF, Thoene JG, et al. Cysteamine therapy for children with nephropathic cystinosis. N Engl J Med 1987;316:971-977.

27. Cohen P, Rogol AD, Deal CL, et al.; 2007 ISS Consensus Workshop participants. Consensus statement on the diagnosis and treatment of children with idiopathic short stature: a summary of the Growth Hormone Research Society, the Lawson Wilkins Pediatric Endocrine Society, and the European Society for Paediatric Endocrinology Workshop. J Clin Endocrinol Metab 2008;93:42104217.

28. Burt RW, Barthel JS, Dunn KB, et al.; NCCN. NCCN clinical practice guidelines in oncology. Colorectal cancer screening. J Nat/ Compr Canc Netw 2010;8:8-61.

29. VHL Family Alliance. The VHL Handbook (fourth edn). http://vhl.org/wordpress/ library/Handbook/handbook40.pdf. Accessed 2 October 2013.

30. Baujat G, Rio M, Rossignol S, et al. Paradoxical NSD1 mutations in BeckwithWiedemann syndrome and 11p15 anomalies in Sotos syndrome. Am J Hum Genet 2004;74:715-720.

31. Terada Y, Imoto I, Nagai H, et al. An 8-cM interstitial deletion on 4q21-q22 in DNA from an infant with hepatoblastoma overlaps with a commonly deleted region in adult liver cancers. Am J Med Genet 2001;103:176-180.

32. Birsner ML, Hoover-Fong J, Bytyci Telegrafi A, Hueppchen NA. Perinatal detection of familial adenomatous polyposis. Obstet Gynecol 2012;120(2 Pt 2):500-503.

33. McDonald-McGinn D, Emanuel B, Zackai E. 22q11.2 Deletion syndrome. http:// www.ncbi.nlm.nih.gov/books/NBK1523/. Accessed 2 October 2013. 